\title{
MANEJO DE LA INFECCIÓN DE VÍAS URINARIAS MULTIRRESISTENTE EN PEDIATRIA
}

\author{
MULTIDRUG RESISTANT MANAGEMENT OF URINARY TRACT \\ INFECTIONS IN PEDIATRICS
}

\author{
Brochet-Bayona Carlos ${ }^{1}$ \\ Pinzón-Consuegra Jorge ${ }^{2}$ \\ Aguilar-Schotborgh Miguel $^{3}$
}

Correspondencias: cjbrochet@yahoo.es

Recibido para evaluación: marzo - 06 - 2015. Aceptado para publicación: octubre - 25 - 2015

\section{RESUMEN}

Introducción: la infección de vías urinarias (IVU) constituye un problema clínico común en los hospitales pediátricos, representa una de las enfermedades más frecuentes en la infancia, su diagnóstico se fundamenta en el cultivo de orina, donde el principal patógeno aislado es Escherichia coli. Convencionalmente, la decisión de manejo se basa en cuatro factores: la bacteria y su sensibilidad, la localización de la infección, el estado clínico y la edad del paciente. Sin embargo, este tratamiento debe iniciarse mucho antes de conocer el germen y su perfil de susceptibilidad. La creciente incidencia de patógenos gramnegativos, resistentes a los fármacos antimicrobianos comunes ha llevado a serias dificultades en relación a su tratamiento, dejando a un lado el uso de antibióticos clásicos o de primera línea, ante la sospecha de multirresistencia.

Objetivo: realizar revisión temática para identificar conceptos referentes a mecanismos de resistencia de los gérmenes gramnegativos, especie de gérmenes multirresistentes y terapéuticas para IVU multirresistente.

Metodología: se efectuó revisión en las bases de datos MEDLINE (1990 a mayo de 2015), CLINICAL KEYS (2001 a marzo 2015), PUBMED (sin fecha límite) y en las listas de referencias de los artículos. La búsqueda bibliográfica arrojó 43 posibles trabajos de investigación, de los cuales se tuvieron en cuenta veinte para desarrollar la presente revisión. Selección a conveniencia.

Resultados: los carbapenémicos como el imipenem, meropenem y ertapenem se convierten en la primera línea para el tratamiento de infecciones debidas a bacterias productoras de betalactamasas de espectro extendido (BLEE). Sin embargo, se ha visto que las quinolonas y los aminoglicosidos pueden mostrar resultados comparables. Desafortunadamente, la resistencia a estos grupos es mayor.

Conclusión: con el pasar del tiempo, las bacterias gramnegativas han logrado expresar nuevos y más eficaces mecanismos de resistencia, conllevando a la aparición de IVUs cada vez más resistentes a la terapia convencional. Por lo que es importante individualizar el manejo de cada paciente según la epidemiología local de cada población. Rev.cienc. biomed. 2015;6(2):340-347

1 Médico. Especialista en Pediatría. Docente Departamento de Pediatría. Grupo de Investigación Niños Heroicos. Facultad de Medicina. Universidad de Cartagena. Cartagena. Colombia.

2 Médico. Estudiante de Postgrado. Pediatría. Grupo de Investigación Niños Heroicos. Facultad de Medicina. Universidad de Cartagena. Cartagena. Colombia.

3 Estudiante de pregrado. Grupo de Investigación Centro de Investigaciones Biomédicas. Facultad de Medicina. Universidad de Cartagena. Cartagena. Colombia. 


\section{PALABRAS CLAVE}

Infecciones urinarias; Pediatría; Bacilos gramnegativos; Farmacorresistencia bacteriana múltiple; Beta-lactamasas.

\section{SUMMARY}

Introduction: urinary tract infection (UTI) is a common clinical problem in pediatric hospitals that represents one of the most common diseases in childhood; diagnosis is based on urine culture, being the Escherichia coli the main isolated pathogen. Conventionally, the management decision is based on four factors: the bacteria and their sensitivity, localization of infection, clinical status and age of the patient, however, this treatment should begin long before knowing the germ and its susceptibility profile. The increasing incidence of gram-negative pathogens resistant to common antimicrobial drugs have led serious difficulties with regard to their treatment, leaving aside the use of classical antibiotics or first line, suspecting multiresistance.

Objective: to make a systematic review in order to identify concepts related to the mechanism of resistance in various gram-negative germs, species of multi resistant germs and treatments for multiresistant UTI.

Material and methods: the review was conducted on the basis MEDLINE (1990 to May 2015), CLINICAL KEYS (2001 to March 2015), PUBMED (no deadline) data and reference lists of articles. The bibliographic search showed 43 possible research papers, of which 20 were taken into account to develop this review.

Results: carbapenems such as imipenem, meropenem and ertapenem become the first line treatment of infections due to bacteria producing beta-lactamases of extended spectrum (ESBL). However, it has been seen that quinolones and aminoglycosides can show comparable results. Unfortunately, the resistance of these groups is higher.

Conclusion: as time goes by, gram-negative bacteria have developed new and more efficient ways of resistance, therefore the emergence of new forms of UTI are more resistant to conventional therapy. That is the reason why the treatment should be individualized according to the local epidemiology of each population. Rev.cienc. biomed. 2015;6(2):340-347

\section{KEYWORDS}

Urinary Tract Infections; Pediatrics; Gram-Negative rods; Drug resistance; Multiple, Bacterial; Bacteria beta-Lactamases.

\section{INTRODUCCIÓN}

La infección de vías urinarias (IVU) constituye un problema clínico común en los escenarios de competencia pediátrica; representa una de las enfermedades más frecuentes en la infancia, ocupando el tercer lugar detrás de las infecciones del tracto respiratorio y gastrointestinal (1). Por lo cual, es una de las infecciones más comunes adquiridas en la comunidad (2), y es considerada, según algunas series, la principal de origen bacteriano (1).

Tal es la importancia que se le adjudica que hacia los siete años de edad $8.4 \%$ de las niñas y $1.7 \%$ de los niños habrán tenido por lo menos un episodio de infección de vías urinarias (3). Algunos autores muestran que la mayor incidencia de infección del tracto urinario se presenta durante el primer año de vida para todos los niños, siendo más prevalente en hombres, en relación a la ausencia de circuncisión, y disminuye sustancialmente para ambos sexos luego del primer año de edad. Por ello, es más significativa esta reducción en varones, logrando una relación niña/niño superior a 10:1 (2-4).

Los estudios de tamizaje en niños febriles menores de dos años que consultan al servicio de urgencias sugieren una prevalencia del 2 al 5\% de infección urinaria como etiología del proceso (4). Un diagnóstico y tratamiento oportuno, además de investigaciones pertinentes para descubrir la presencia de alteraciones en el riñón o las vías urinarias ayudarán a disminuir la morbilidad por complicaciones agudas como septicemia, a mediano o a largo plazo cicatrices renales, hipertensión arterial, preeclampsia, microalbuminuria y enfermedad renal crónica (1,4$6)$. De ahí el interés por establecer guías para la identificación temprana, y tratamiento oportuno y eficaz. 
El tracto urinario por definición es estéril en condiciones normales, si se hace la salvedad del período neonatal, la mayoría de las infecciones bacterianas ocurren vía ascendente tras la colonización por gérmenes intestinales del epitelio periuretral, uretral y vesical que pueden alcanzar el tejido renal desde el uréter (7).

En niños, el diagnóstico se fundamenta en el cultivo de orina, siendo el principal patógeno aislado Escherichia coli (76 - 90\%) seguido por Klebsiella pneumoniae $(0,6-8 \%)$, Proteus mirabilis (0.5 - 6\%) Pseudomonas (2\%) Staphyloccocus spp. (1-5\%) $(1,3)$. Convencionalmente, la decisión de manejo se basa en cuatro factores que influyen en la elección del antimicrobiano: la bacteria y su sensibilidad, la localización de la infección, el estado clínico y la edad del paciente; los objetivos de esta terapia son erradicar el germen, evitar complicaciones y el daño renal a futuro.

Por lo que la recomendación general es que una vez se sospeche la presencia de IVU por datos clínicos y paraclínicos se debe iniciar tratamiento sin esperar resultado de urocultivo $(2,6,8,9)$. Como deja entrever esta sentencia, la recomendación implica iniciar tratamiento mucho antes de conocer el germen y su perfil de susceptibilidad, por lo que la antibioticoterapia se instaura de forma empírica con cobertura que incluye gérmenes gramnegativos y basándose en el conocimiento de la epidemiología local $(9,10)$.

Con respecto al tratamiento se han mostrado cada día más informes sobre la resistencia de los gérmenes a los medicamentos tradicionales recomendados para el manejo empírico inicial de la IVU, entre los que figuran el trimetoprim, la ampicilina y las cefalosporinas de primera generación $(3,8)$. Por otra parte, el uso de medicamentos profilácticos en pacientes pediátricos con alto riesgo de desarrollar cicatrices renales por pielonefritis recurrentes ha llevado a cambios en la flora bacteriana hacia gérmenes resistentes (10).

La creciente incidencia de patógenos gramnegativos resistentes a los fármacos antimicrobianos comunes, conlleva a serias dificultades en relación a su tratamiento, no solo a infecciones complicadas, sino además a aquellas adquiridas en la comunidad $(3,4)$. En estas bacterias, el principal mecanismo de resistencia es la producción de betalactamasas; en los últimos estudios, se ha establecido que estos microorganismos productores de betalactamasas de espectro extendido (BLEE) se distribuyen en todo el mundo $(11,12)$.

Lo que convierte a estas nuevas IVU multirresistentes en un problema de salud pública a nivel mundial, y de ahí la importancia de desarrollar una revisión temática que permita realizar un acercamiento a los conceptos referentes a mecanismos de resistencia de los gérmenes gramnegativos, especie de gérmenes multirresistentes y terapéuticas para IVU multirresistente.

\section{MATERIALES Y MÉTODOS}

Tipo de estudio: se efectuó una revisión de tema sobre el manejo de las infecciones de las vías urinarias multirresistentes, se condensaron los hallazgos y las conclusiones de los diferentes artículos con el fin de comprender mejor los resultados encontrados.

Estrategia de búsqueda bibliográfica: en esta revisión, se realizaron búsquedas en las bases de datos MEDLINE (1990 a mayo de 2015), CLINICAL KEYS (2001 a marzo 2015), PUBMED (sin fecha límite) y en las listas de referencias de los artículos.

Términos de búsqueda: se utilizaron para la revisión los siguientes descriptores DeCS: "infecciones urinarias" "pediatría" "farmacorresistencia bacteriana múltiple", así como su respectiva traducción al inglés.

Metodología de revisión de los artícuIos: se estudiaron los títulos, resúmenes de los documentos seleccionados en las bases de datos consideradas, si se ajustaban a las exigencias del objetivo planteado se trataba de obtener los artículos completos. Se consideraron artículos completos a conveniencia.

\section{RESULTADOS}

Se seleccionaron un total de 43 artículos, teniendo en cuenta los criterios mencionados 
anteriormente. De estos se excluyeron 23 por usar subpoblaciones pediátricas, hablar del manejo de infecciones de vías urinarias multirresistentes en contextos específicos, y por no delimitar claramente sus métodos de identificación de resistencia antimicrobiana. Selección a conveniencia.

\section{Mecanismos de resistencia de gérmenes gramnegativos}

Recientemente, el uso indiscriminado e irracional de los antibióticos y el desconocimiento de los mecanismos de resistencia bacteriana han conducido a la limitación en las opciones terapéuticas frente a microorganismos causales de infecciones comunes $(3,4)$. Teniendo en cuenta que las bacterias gramnegativas tienen una variedad de mecanismos de resistencia y que la selección de estos mecanismos puede llevar a fallas terapéuticas, es necesario conocer los más prevalentes.

La resistencia bacteriana a antimicrobianos puede ser natural o adquirida, y esta última puede aparecer por diferentes mecanismos, en su mayoría genéticos. Algunas de estas alteraciones ocurren espontáneamente por pequeños cambios a nivel de algunos pares de nucleótidos que codifican para grandes segmentos del material genético bacteriano, tales como: inversiones, duplicaciones, inserciones o transposiciones de secuencias de ADN, de un sitio a otro del cromosoma bacteriano, mientras otras bacterias adquieren esta información por transferencia de secuencias de ADN a través de plásmidos y transposones $(13,14)$.

Con esta nueva información las bacterias expresan sus mecanismos de resistencia principalmente por medio de cuatro formas: enzimas inactivantes, alteración del sitio receptor, alteración de la permeabilidad de la membrana y por eflujo activo de antibióticos (13). Los bacilos gramnegativos expresan con mayor frecuencia el mecanismo de resistencia ligado a la producción de enzimas, dentro de las cuales las betalactamasas se han convertido en las más estudiadas $(14,15)$.

Estas actúan rompiendo el anillo betalactámico por medio de un mecanismo de hidroli- sis, inactivando de esa forma los antibióticos de este grupo. Se han clasificado históricamente en cuatro grupos (1, 2, 3 y 4$)$ según su perfil de inhibición, y en cuatro categorías según su clase molecular ( $A, B, C$ y D) (15), tal como se describe en la Tabla No 1 . Cabe resaltar un grupo de betalactamasas con un espectro de acción más amplio, llamadas betalactamasas de espectro extendido (BLEE), las cuales son principalmente expresadas por cepas de Klebsiella spp. y Escherichia coli (16), estas enzimas son particularmente resistentes a la acción de cefalosporinas de tercera generación y monobactámicos.

\begin{tabular}{l}
\hline \multicolumn{4}{|c|}{ TABLA No 1.} \\
CLASIFICACIÓN DE BETALACTAMASAS \\
SEGÚN CLASE MOLECULAR Y PERFIL DE \\
INHIBICIÓN \\
Grupo
\end{tabular}

Finalmente tenemos otro grupo de enzimas cuya acción hidroliza la estructura molecular de los carbapenemas (1) y las carbapenemasas, de estas enzimas se han descrito dos familias: las carbapenemasas de serina identificadas principalmente en enterobacterias y en Acinetobacter spp., y las metalobetalactamasas encontradas principalmente en especies como Serratia, Klebsiella, Enterobacter cloacae, Citrobacter freundii y E. coli (17).

En cuanto a los otros mecanismos de resistencia, las bacterias logran la disminución de la concentración del antimicrobiano en su interior gracias a las bombas de flujo y el cambio en la permeabilidad de la membrana a través del cierre o la pérdida de porinas. Las 
bombas de flujo utilizan la hidrolisis del ATP no solo para la expulsión de antimicrobianos, sino también para la expulsión de gran número de materiales tóxicos, metabolitos, solventes orgánicos entre otras sustancias que potencialmente tienen acción bactericida. Estas bombas se clasifican en seis familias: $A B C$ (ATP binding cas-sette), MF (major facilitator), MATE (multidrug and toxic efflux), RND (resistance nodulation division), SMR (small multidrug resistance) y DMT (drug/metabolite transporter superfa-mily).

Por otra parte, las porinas, canales de la membrana externa de las bacterias gramnegativas (BGN), trabajan como filtros permitiendo el paso de antibióticos. Las BGN pueden alterar este mecanismo, a través de mutaciones específicas que llevan al cierre o no expresión de las porinas e impidiendo la entrada de estos fármacos $(13,14,17)$. Por último, la alteración del sitio receptor como ocurre en el cambio de las proteínas de unión a penicilinas, blanco de acción de los antibióticos ß-lactaminos.

\section{Infección de vías urinarias por gérme- nes multirresistentes}

En la actualidad, las IVU por gérmenes multirresistentes están en aumento. La sensibilidad a los antibióticos de los principales gérmenes aislados ( $E$. coli, $P$. mirabilis $y K$. pneumoniae) evidencian una pérdida in vitro a los antibióticos considerados de primera línea terapéutica (4).

En un estudio realizado en la población pediátrica brasileña se evidenció que la nitrofurantoína, usada como medicamento de primera línea de instauración oral, ha mostrado a lo largo de un período considerable de tiempo mantener buena actividad contra el E. coli, a diferencia de otros medicamentos considerados de primera línea como trimetoprim-sulfa y ampicilina; sin embargo, se demostró su pobre efectividad en casos de IVU febriles o en casos donde se sospecha compromiso renal, dado que no alcanzan concentraciones terapéuticas en sangre para tratamiento de estos casos.

Se recomienda a la nitrofurantoína como opción para tratamiento de IVU afebriles e in- cluso en profilaxis cuando así es requerido (8). De forma similar, los datos reportados en un estudio sobre la población pediátrica en Cali comprobaron la alta resistencia a los medicamentos utilizados de primera línea como sulfas, ampicilina y cefalosporinas de primera generación, a diferencia de lo observado con nitrofurantoína en la que se evidenciaba adecuada acción y sensibilidad (3).

Similares características fueron encontradas en pacientes de Medellín, en los que se encontró poca resistencia a la nitrofurantoína (18). Lo anterior contrasta con otro estudio realizado en una población pediátrica de Medellín, en donde el patrón de sensibilidad para $E$. coli muestra, según lo reportado, una alta resistencia a nitrofurantoína, así como otros antibióticos considerados de primera elección como las cefalosporinas de primera generación, trimetoprim-sulfametoxasol, ampicilina, ampicilina-sulbactam, amoxicilina y amoxicilina-ácido clavulónico (4).

Por otra parte, distintos autores coinciden en que la sensibilidad a aminoglucósidos permanece estable, instaurando estos como medida terapéutica inicial y segura en IVU afebriles, estos seguramente no impactan negativamente en la flora intestinal, de tal forma que la tasa de resistencia se mantiene muy baja $(3,8,18)$.

Además de lo anterior, al utilizarse como tratamiento empírico inicial en pacientes con infecciones febriles se demostró en un estudio que el $87.4 \%$ se encuentran afebriles a las 36 horas y el $100 \%$, a las 72 horas de iniciado el antibiótico. Independientemente del perfil de resistencia, lo anterior junto con la sensibilidad mostrada en su población de estudio mayor del $95 \%$ a la amikacina y la respuesta clínica favorable al tratamiento, hacen que se concluya en el uso de aminoglucósidos para manejo de IVU febril (4). La recomendación entonces para el uso seguro de aminoglucósidos es evaluar la función renal y de ser posible, indicar antibiótico en una dosis diaria de preferencia endovenosa $(4,6)$.

Con respecto a otros medicamentos para manejo de IVU febriles se ha reportado baja resistencia de $E$. coli a cefalosporinas de 
tercera generación (3-5\%) (8), también se evidencia adecuada sensibilidad (mayor del $80 \%$ ) a aminoglucósidos, cefalosporinas de segunda y tercera generación y quinolonas (3). Además de esto, también se ha evidenciado que las cefalosporinas de tercera y cuarta generación pueden ser un posible medicamento de elección en IVU complicadas a la luz del perfil de resistencia bajo, cosa que reportaría resistencia del E. coli a ceftazidima y cefepime en menos del $4 \%$.

No obstante, no se debe olvidar el alto riesgo de inducir la producción de BLEE reportado en $6.3 \%$ de infecciones por E. coli (18); en cuyo caso, la elección del antibiótico se dirigiría a escoger carbapenémicos, aunque la bacteria muestra sensibilidad a quinolonas o aminoglucósidos, la respuesta clínica suele ser comparable cuando se utilizan estos últimos (19).

Finalmente, Ilama la atención que el reporte realizado por Hoyos et al. en una población pediátrica de la ciudad de Medellin, en donde al evaluar los patrones de resistencia en los aislamientos considerados como adquiridos en la comunidad se resaltó la presencia de bacterias productoras de BLEE y betalactamasas de tipo AmpC, que tradicionalmente se habían considerado de origen hospitalario $(1.85 \%$ y $11.3 \%$, respectivamente).

Las bacterias $E$. coli y $K$. pneumoniae fueron las bacterias productoras de BLEE y la Citrobacter freundii y $E$. cloacae fueron las bacterias con mayor producción de betalactamasa de tipo AmpC. En el estudio también se reportó que en cuatro aislamientos de $E$. coli y en cuatro de $K$. pneumoniae también hubo un patrón de resistencia sugestivo de betalactamasa de tipo AmpC, lo cual sugiere que por ser naturalmente transferibles facilitan la diseminación de la resistencia mediada por este gen (4).

\section{Manejo de IVU multirresistentes}

Se debe tener en cuenta que la terapia antibiótica empírica inicial que se adoptará como manejo del paciente siempre debe obedecer a la epidemiología de cada institución de salud. Esto implica necesariamente la necesidad de desarrollar estudios y seguimientos epidemiológicos y estadísticos que permitan tener amplio conocimiento sobre los comportamientos de las infecciones y su respuesta a los grupos antibióticos disponibles e indicados para cada una de las situaciones clínicas. Se resalta, además, la importancia de compartir y difundir la información obtenida con el objetivo de evaluar la resistencia bacteriana y proponer medidas de impacto que busquen erradicarla en caso de existir (1).

Es de vital importancia revisar la epidemiología y susceptibilidad local, ya que estudios de diferentes ciudades muestran que las sensibilidades a ciertos compuestos pueden variar entre las ciudades de un mismo país, como es el ejemplo de sensibilidad a amoxicilina-ácido clavulánico que muestra diferencias significativas entre Bogotá (91\%) y Cali $(63 \%)(3)$.

Por esta razón se recomienda revisar y conocer la susceptibilidad in vitro de los principales agentes uropatógenos del centro donde se labora, más que seguir las sugerencias de publicaciones foráneas (9). Sin embargo, teniendo como base los estudios de sensibilidad de locaciones cercanas, valdrá la pena evaluar la sensibilidad de la nitrofurantoína en instancia inicial como medicación para manejo de infecciones febriles o con alto riesgo de compromiso renal, valorar los aminoglucosidos, cefalosporinas de segunda y tercera generación, quinolonas y las fluoroquinolonas, estas últimas de uso muy controvertido en pediatría por su riesgo de toxicidad articular.

Las fluoroquinolonas constituyen un agente antimicrobiano potencial de primera línea en pielonefritis e IVU complicadas. Son usadas generalmente en situaciones en que los antimicrobianos usualmente recomendados no son apropiados en base a su susceptibilidad in vitro o antecedente de eventos adversos. De tal manera que la Academia Americana de Pediatría actualmente se apoya en el uso de la ciprofloxacina como terapia oral de las IVU causadas por $P$. aeruginosa u otras bacterias gramnegativas multirresistentes en niños entre 1 y 17 años de edad (9). 
Las penicilinas constituyen otra opción de manejo cuando están unidas a inhibidores de betalactamasas. Con respecto a la amoxicilina-clavulonato, no figura en las últimas guías de la Infectious Diseases Society of America (IDSA) sobre las opciones para el tratamiento de la pielonefritis aguda, pero podría convertirse en una alternativa oral para el manejo ambulatorio dirigido en bacterias sensibles a este compuesto.

En el caso de piperacilina-tazobactam estudios recientes demuestran equivalencia con imipenem en pielonefritis (20), de manera que, ante la sospecha de infección por bacterias distintas de $E$. coli o multirresistentes, se considera una opción empírica aceptable.

El aumento de incidencia de infecciones por uropatógenos productores de BLEE obliga a pensar en otras opciones terapéuticas, una de las alternativas para pacientes con pielonefritis con factores de riesgo de infección por BLEE que no se encuentren en sepsis grave o en shock séptico es el ertapenem. Los carbapenémicos como el imipenem, meropenem y ertapenem se convierten en la primera línea para el tratamiento de infecciones debidas a bacterias productoras de BLEE; sin embargo, se ha visto que las quinolonas y los aminoglucósidos pueden mostrar resultados comparables (16).

\section{Recomendaciones}

Según los resultados que arrojen los estudios en cada institución, la recomendación en la elección de medicamentos en IVU en niños se basaría en el porcentaje de resistencia, siguiendo un patrón similar al ofrecido por Castaño et al. (3).

[A] Para el manejo de cistitis y pielonefritis aguda NO elegir compuestos con resistencia bacteriana mayor de 50\%: ampicilina, trimetoprim-sulfa, cefalexina, ampicilina-sulbactam. [B] Para el manejo de cistitis aguda elegir antibióticos con resistencia bacteriana moderada entre 30\% y $50 \%$ (sensibilidad 50-80\%): amoxicilina, amoxicilina-clavulánico, cefaclor, cefadroxilo, cefradina y cefazolina. [C] Para el manejo de pielonefritis aguda elegir medi- camentos con sensibilidad mayor de $80 \%$ : aminoglucósidos, ceftriaxona, cefuroxime, cefprozil, cefixime. [D] A pesar que el ácido nalidíxico y la nitrofurantoína mostraron baja resistencia bacteriana ( $5 \%$ y $9 \%$ respectivamente) se recomiendan como medicamentos de primera línea solo en cistitis aguda. No se recomiendan para manejo de pielonefritis aguda.

Finalmente, si se ignora este proceso de resistencias emergentes a gran velocidad, es posible que la comunidad médica se vea obligada a usar carbapenémicos como primera opción en el manejo empírico de IVU severas originadas desde la comunidad (19), cosa que explica el uso consciente y medido de antibióticos. Asimismo conocer los perfiles de resistencia de la comunidad con la que se trabaja para intentar disminuir el proceso de selección de bacterias resistentes.

\section{CONCLUSIÓN}

En estos últimos años se ha evidenciado el comportamiento "pensante" de estos microorganismos capaces de modificar su estructura genómica para colonizar y causar enfermedad en las poblaciones. Estos mecanismos cada vez son más variados y eficaces, y dificultan la elección empírica y específica del antibiótico.

Cada año, aumentan los reportes de nuevas cepas resistentes en diferentes lugares del mundo, obligando a investigar $y$ producir nuevos fármacos con mayores coberturas. Por lo que es imperativo que cada médico conozca su epidemiología y que individualice la elección del antibiótico, evitando de esa forma la selección de cepas cada vez más resistentes que nos lleven a una realidad sin opciones, en donde más niños presenten IVUs multirresistentes, que en el peor de los casos provoquen su muerte.

CONFLICTOS DE INTERESES: ninguno que declarar.

FINANCIACIÓN: recursos propios de los autores. 


\section{REFERENCIAS BIBLIOGRÁFICAS}

1. Mendoza Pertuz J, Colmenares Mart A, Montero Carvajalino AE. Enfoque diagnóstico y terapéutico del primer episodio de infección del tracto urinario en pediatría. CCAP. 2013;12(3):5873.

2. Stein R, Dogan HS, Hoebeke P, Kocvara R, Nijman R, Radmayr C, et al. Urinary tract infections in children: EAU/ESPU guidelines. European urology. 2015;67(3):546-58.

3. de Castaño I, González C, Buitrago ZY, de Rovetto C. Etiology and bacterial sensibility in children urinary infection. Hospital Infantil Club Noel and Hospital Universitario del Valle, Cali, Colombia2009.

4. Hoyos Á, Serna L, Ortiz G, Aguirre J. Infección urinaria adquirida en la comunidad en pacientes pediátricos: clínica, factores de riesgo, etiología, resistencia a los antibióticos y respuesta a la terapia empírica. Infectio. 2012;16:94-103.

5. Gonzalo de Liria C, Méndez Hernández M, Azuara Robles M. Infección urinaria. In: ERGON, editor. Protocolos diagnostico-terapeuticos de infectologia. 3ra ed2011.

6. Salas del C P, Barrera B P, González C C, Zambrano O P, Salgado D I, Quiroz L, et al. Actualización en el diagnóstico y manejo de la infección urinaria en pediatría. Revista chilena de pediatría. 2012;83:269-78.

7. Martínez Suárez V, Santos Rodríguez F. Infección de las vías urinarias (ITU) en el niño: Plan diagnóstico-terapéutico. BOL PEDIATR. 2006;46(2):222-9.

8. Guidoni EBM, Berezin EN, Nigro S, Santiago NA, Benini V, Toporovski J. Antibiotic resistance patterns of pediatric community-acquired urinary infections. Brazilian Journal of Infectious Diseases. 2008;12:321-3.

9. Cavagnaro F. Infección urinaria en pediatría: Controversias. Revista Chilena de Infectología. 2012;29:427-33.

10. Nateghian AR, Robinson JL, Mohandessi S, Hooman N. Resistance pattern of breakthrough urinary tract infections in children on antibiotic prophylaxis. Journal of infection and public health. 2009;2(3):147-52.

11. Bitsori M, Maraki S, Kalmanti M, Galanakis E. Resistance against broad-spectrum beta-lactams among uropathogens in children. Pediatric nephrology (Berlin, Germany). 2009;24(12):23816.

12. Giamarellou H. Multidrug resistance in Gram-negative bacteria that produce extended-spectrum beta-lactamases (ESBLs). Clinical microbiology and infection : the official publication of the European Society of Clinical Microbiology and Infectious Diseases. 2005;11 Suppl 4:1-16.

13. Daza-Pérez RM. Resistencia bacteriana a antimicrobianos: Su importancia en la toma de decisiones en la práctica diaria. Información Terapéutica del Sistema Nacional de Salud. 1998;22(3):57 - 67.

14. Martin N G. Resistencia bacteriana a B-lactámicos: Evolución y mecanismos. Archivos Venezolanos de Farmacología y Terapéutica. 2002;21:107-16.

15. Abarca G, Herrera ML. Betalactamasas: Su importancia en la clínica y su detección en el laboratorio. Revista Médica del Hospital Nacional de Niños Doctor Carlos Sáenz Herrera. 2001;36:77-104.

16. Hernandez W, Ramo A, Nodarse R, Padrón A, De Armas E. Resistencia bacteriana en las bacterias productoras de betalactamasas extendidas (BLEE). Rev.Cub.Med.Int.Emerg. 2006;5(1):256 - 64

17. Tafur JD, Torres JA, Villegas MV. Mecanismos de resistencia a los antibióticos en bacterias Gram negativas. Infectio. 2008;12:227-32.

18. Vélez Echeverri CM, Serna-Higuita LM, Serrano Gayubo AK, Ochoa-García C, Rojas Rosas L, Bedoya AM, et al. Resistance profile for pathogens causing urinary tract infection in a pediatric population, and antibiotic treatment response, at a university hospital 2010-20112014.

19. Pitout JDD. Recent changes in the epidemiology and management of extended-spectrum $\beta$-lactamase-producing Enterobacteriaceae. F1000 Medicine Reports. 2009;1.

20. Bisso Andrade A. Antibioticoterapia en las infecciones graves. Acta Médica Peruana. $2011 ; 28: 27-38$.

Universidad de Cartagena Fundada en 1827

REVISTA,CIENCIAS BIOMÉDICAS
Es el órgano de información científica de la Facultad de Medicina de la Universidad de Cartagena. Colombia.

Publique su trabajo en esta revista enviando su manuscrito a: revistacienciasbiomedicas@unicartagena.edu.co www.revistacienciasbiomedicas.com www.revistacienciasbiomedicas.com.co

Revista ciencias Biomédicas es una publicación independiente, imparcial, abierta, revisada por pares, de elevada visibilidad internacional, con circulación online e impresa. Publica artículos en todas las modalidades universalmente aceptadas en inglés y en español, de temas referentes con todas las ciencias biomédicas, incluyendo ámbitos clínicos, epidemiológicos o de estudios básicos.

El sistema de gestión de manuscritos es rápido y justo.

Revista Ciencias Biomédicas está incluida en varias bases de datos latinoamericanas e internacionales.

Antes de enviar su manuscrito, revise las recomendaciones para los autores, presentes en: www.revistacienciasbiomedicas.com www.revistacienciasbiomedicas.com.co 